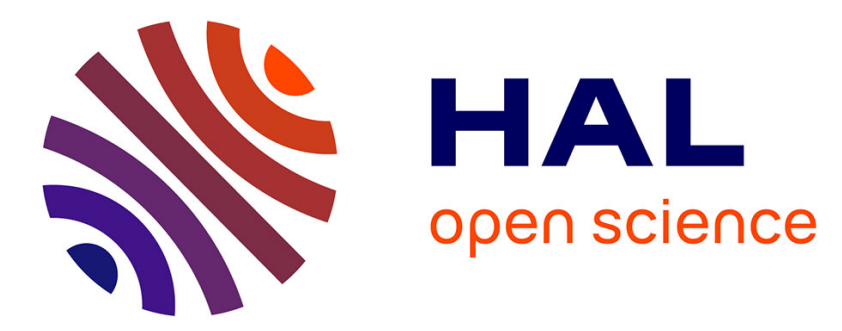

\title{
Croissance estivale en matière sèche de peuplements de fétuque élevée (Festuca arundinacea Schreb.) et de dactyle (Dactylis glomerata L.) dans l'Ouest de la
} France. II. Interaction entre les niveaux d'alimentation hydrique et de nutrition azotée

\author{
Gilles Lemaire, Alain Denoix
}

\section{To cite this version:}

Gilles Lemaire, Alain Denoix. Croissance estivale en matière sèche de peuplements de fétuque élevée (Festuca arundinacea Schreb.) et de dactyle (Dactylis glomerata L.) dans l'Ouest de la France. II. Interaction entre les niveaux d'alimentation hydrique et de nutrition azotée. Agronomie, 1987, 7 (6), pp.381-389. hal-00885005

\section{HAL Id: hal-00885005 https://hal.science/hal-00885005}

Submitted on 1 Jan 1987

HAL is a multi-disciplinary open access archive for the deposit and dissemination of scientific research documents, whether they are published or not. The documents may come from teaching and research institutions in France or abroad, or from public or private research centers.
L'archive ouverte pluridisciplinaire HAL, est destinée au dépôt et à la diffusion de documents scientifiques de niveau recherche, publiés ou non, émanant des établissements d'enseignement et de recherche français ou étrangers, des laboratoires publics ou privés. 


\section{Croissance estivale en matière sèche de peuple- ments de fétuque élevée (Festuca arundinacea Schreb.) et de dactyle (Dactylis glomerata L.) dans l'Ouest de la France. II. Interaction entre les niveaux d'alimentation hydrique et de nutri- tion azotée}

Gilles LEMAIRE \& Alain DENOIX $\left({ }^{*}\right)$

I.N.R.A. Station d'Ecophysiologie des Plantes fourragères, Centre de Recherches de Lusignan, $F 86600$ Lusignan

(*) Station d'Agronomie, Centre de Recherches d'Angers, $F 49000$ Angers

FNIE, 58, avenue Kléber, F 75784 Paris

Un article précédent avait permis d'étudier la croissance potentielle estivale de peuplements de fétuque élevée et de dactyle, lorsque ni l'alimentation hydrique ni la nutrition azotée n'étaient limitantes.

Dans ce présent article, nous avons étudié comment une restriction simultanée des disponibilités en eau et en azote réduisait la croissance en matière sèche.

Pour cela, nous avons suivi la dynamique de croissance de différentes parcelles en situation sèche ou irriguée avec différents niveaux d'apport d'azote, en mesurant simultanément les quantités d'azote prélevées et les quantités d'eau consommées.

L'analyse simultanée de ces différentes cinétiques nous a permis de mettre en évidence les mécanismes de l'interaction entre alimentation hydrique et nutrition minérale ainsi que ses conséquences sur la croissance en matière sèche.

Le dessèchement des horizons superficiels du sol provoque une très nette diminution de la disponibilité en azote pour la plante et, très rapidement, l'effet direct du stress hydrique est amplifié par une diminution du niveau réel de nutrition azotée. Ceci peut être diagnostiqué par une déformation des courbes de dilution $\mathrm{N} \%=\alpha(\mathrm{MS})^{-\beta}$. Les résultats analysés ont permis de montrer que ce phénomène pouvait être amplifié ou bien atténué selon le régime pluviométrique, qui détermine le mode de dessèchement des différents horizons du profil, et la nature de l'enracinement de l'espèce consiaerée. Le dactyle semble à cet égard moins sensible que la fétuque et il manifeste une plus grande aptitude à extraire l'azote du sol dans les horizons superficiels.

Ce phénomène, déjà observé sur d'autres cultures, prend une importance considérable pour les prairies où les éléments fertilisants apportés en couverture sont essentiellement localisés dans les horizons très superficiels du sol.

Mots clés additionnels : Courbes de croissance, consommation d'eau, efficience de l'eau, évapotranspiration réelle, évapotranspiration potentielle. water consumption and nitrogen nutrition.

In a previous paper, we studied the potential summer regrowth of tall fescue and cocksfoot in conditions where neither water nor nitrogen were limiting. In this paper, we studied the effect of simultaneous shortage of water and nitrogen on dry matter production, by examining the growth dynamics of different plots under irrigated or non-irrigated conditions, with differents levels of nitrogen application. Nitrogen uptake and water consumption were simultaneously recorded. Analysis of these kinetics during summer regrowth allowed us to determine the mechanisms of water and nitrogen interaction in field conditions and its effects on dry matter production. Drying of the upper layers of the soil rapidly limited nitrogen availability for the plants. The direct effect of water stress was amplified by a shortage of nitrogen. This phenomenon could clearly be illustrated with dilution curves $\mathrm{N} \%=\alpha(\mathrm{DM})^{-\beta}$. Results obtained over five years showed that this phenomenon could be amplified or reduced according to the root system of the grass species. Cocksfoot appeared to be less susceptible to drying of the first 20 centimeters of soil than tall fescue and had a greater capacity for extracting nitrogen from the soil. The frequency of rewatering of soil surface by some might rainfall also plays a large role in the availability of nitrogen in dry conditions. This phenomenon has been already observed on annual crops but it is important for grassland because fertilizers are usually top-dressed and remain located in the upper layers of the soil.

Additional key words: Growth curves, water consumption, water consumption efficiency, actual evapotranspiration, potential evapotranspiration. 


\section{INTRODUCTION}

Dans un article précédent (LEMAIRE \& DENOIX, 1987) nous avons montré que l'on pouvait déterminer le potentiel de croissance en matière sèche d'un peuplement de graminées fourragères lorsque à la fois le niveau d'alimentation hydrique et le niveau de nutrition azotée étaient non limitants.

Dans les conditions réelles de croissance, en période estivale, ces 2 facteurs se trouvent rarement simultanément à un niveau optimum, ils contribuent donc à limiter les capacités de croissance des prairies en été. De plus, leurs effets limitants ne sont pas seulement additifs car il existe une interaction très forte entre eux.

Cette interaction est en général positive et se traduit par une amplification de l'effet de l'azote sur la croissance en matière sèche lorsque le niveau d'alimentation hydrique devient optimum (COLMAN \& LAZENBY, 1975) et réciproquement. Cette constatation, somme toute assez banale, est la résultante de phénomènes multiples qui font que la disponibilité de l'azote dans le sol est en partie dépendante du niveau de disponibilité en eau des horizons supérieurs (GARWOOD \& WILLIAMS, $1967 a$ et $b$ ). D'une manière générale, plusieurs auteurs montrent que le taux de récupération apparent de l'azote apporté augmente avec l'irrigation (SMIKA et al., 1965 ; WHITE \& BROWN, 1972 ; TURNER, 1979). Cependant on ne peut pas déterminer si cela provient de l'augmentation de la capacité de croissance des plantes ou bien s'il s'agit réellement d'une meilleure disponibilité de l'azote liée à la fois à l'état hydrique du sol et/ou à une meilleure exploration racinaire.

D'autre part, il a été montré que l'efficience de l'eau consommée était fortement influencée par le niveau de nutrition azotée (SMIKA et al., 1965; WHITE \& BROWN, 1972). Cette forte augmentation de l'efficience de l'eau consommée est la résultante d'une augmentation importante de la croissance en matière sèche, consécutive à un apport d'azote, alors que la consommation d'eau n'est pas augmentée (SMIKA et al., 1965) ou peu augmentée (BURZLAFF et al., 1968 ; WHITE \& BROWN, 1972 ; LAUENROTH \& SiMS, 1976).

Ainsi, pour pouvoir quantifier l'effet d'une restriction simultanée de l'azote et de l'eau sur la diminution de la capacité de croissance d'une prairie en été, il est nécessaire d'analyser plus précisément les mécanismes par lesquels l'un des facteurs est susceptible d'agir sur la disponibilité de l'autre facteur.

\section{MATÉRIEL ET MÉTHODES}

Le dispositif expérimental a été décrit dans une note précédente (G. LEMAIRE \& A. DENOIX, 1987). Nous rappelons ici que nous disposions de 2 séries d'expérimentations sur le domaine expérimental des Verrines à Lusignan :

- en 1979-80-81 : une analyse de la croissance de la fétuque élevée $\mathrm{cv}$. Clarine et du dactyle cv. Floreal en conditions sèches et en conditions irriguées avec 3 niveaux de nutrition azotée : 0,60 et $120 \mathrm{~kg}$;
- en 1984-85 : sur les mêmes génotypes, en conditions sèches et irriguées avec 2 niveaux de nutrition azotée : 60 et $120 \mathrm{~kg}$.

La cinétique de croissance était suivie pendant 7 à 8 semaines à partir d'une coupe d'homogénéisation fin juin, par des prélèvements hebdomadaires réalisés à la motofaucheuse sur des sous-parcelles élémentaires de $6 \mathrm{~m}^{2}$. Un échantillon d'herbe était prélevé simultanément pour analyse de la teneur en azote total.

Les apports d'eau sur les parcelles irriguées étaient réalisés au moyen d'un dispositif de micro-asperseurs (LEMAIRE \& ROBERGE, 1980). L'homogénéité des apports d'eau était satisfaisante grâce à un maillage très dense $(5 \times 5 \mathrm{~m})$ des asperseurs.

Les apports d'azote étaient effectués sous forme d'ammonitrate immédiatement après la coupe d'homogénéisation et étaient immédiatement suivis d'une irrigation de 5 à $10 \mathrm{~mm}$, sur l'ensemble des parcelles, destinée à dissoudre l'engrais et à assurer son incorporation au sol. Par la suite, les parcelles « en sec » ne recevaient plus aucun apport d'eau d'irrigation. En 1984, cependant, après un printemps exceptionnellement sec, les réserves hydriques du sol étaient épuisées au moment de la coupe de fin juin et un apport d'eau «exceptionnel » de $25 \mathrm{~mm}$ a été réalisé sur les traitements « sec » pour permettre un redémarrage minimum de la croissance.

Les consommations hydriques de chaque traitement étaient suivies par sonde à neutron jusqu'à une profondeur de $1,80 \mathrm{~m}$ avec un tube par répétition ( 5 en $1979-80-81$, et 4 en 1984-85). Nous n'avons jamais observé de variations d'humidité à cette profondeur. Les possibilités de percolation d'eau en profondeur pendant la période estivale sont totalement inexistantes du fait d'une irrigation très bien contrôlée, l'humidité du sol ayant toujours été maintenue inférieure à la capacité au champ. Par contre, on ne peut pas exclure des possibilités de remontées capillaires. Cependant, la nature du matériau et du type de porosité du sous-sol nous laisse penser que celles-ci doivent être négligeables sur de telles distances (CHESSERON, 1986).

Les sondages ètaient effectués chaque semaine, le même jour que les prélèvements pour la détermination de la croissance en matière sèche. De plus, sur les parcelles irriguées, des sondages supplémentaires étaient réalisés juste avant et le lendemain de l'irrigation, ce qui permet de minimiser les hétérogénéités induites par les apports d'eau (Lemaire \& ROBERGE, 1980).

Le sol de Lusignan, localement appelé " terre rouge à châtaigniers ", est un sol brun lessivé sur limon, superposé à une argile rouge à pisolithes. La texture, limoneuse dans les $501^{\text {ers }}$ centimètres, devient argilolimoneuse au-delà, avec apparition de concretions ferro-manganiques et de taches d'oxydo-réduction du fer. Un profil type de ce sol a été décrit par CHESSERON (1986). La disponibilité en eau est importante dans les $1001^{\text {rs }}$ centimètres (R.U. de $2 \mathrm{~mm}$ d'eau par $\mathrm{cm}$ de sol), elle est beaucoup plus faible dans les horizons profonds constitués d'argiles rubéfiées compactes (densité apparente : 1,8 ) où l'exploration racinaire est limitée aux galeries de vers de terre et à quelques fissures verticales. 


\section{RÉSULTATS ET DISCUSSIONS}

\section{A. Analyse des courbes de croissance en matière sèche}

On peut distinguer immédiatement 3 types d'années selon l'intensité de l'effet sécheresse sur la croissance en matière sèche (fig. 1) :

1981 : année à sécheresse faible. L'effet de l'irrigation est pratiquement nul sur la fétuque et très faible sur le dactyle.

1979 et 1980 : années à sécheresse moyenne. L'effet de la sécheresse se traduit sur les 2 espèces par une réduction d'environ $1 \mathrm{tha}^{-1}$ de matière sèche au bout de 50 jours de repousse, soit environ 20 p. 100 . On s'aperçoit que pour ces 2 années, l'effet sécheresse est du même ordre de grandeur que celui d'une réduction de 120 à $60 \mathrm{~kg}$ de l'apport d'azote (superposition exacte des courbes $120 \mathrm{~N}$ sec et $60 \mathrm{~N}$ irrigué pour les 2 espèces). L'effet de l'azote apparaît être sensiblement de la même amplitude en sec qu'en irrigué.

1984 et 1985 : années à sécheresse forte, particulièrement en 1984 où il a été nécessaire de pratiquer des irrigations exceptionnelles sur les parcelles « sec» pour permettre une croissance minimum des plantes. L'effet de la sécheresse se traduit par une diminution très forte de la croissance en matière sèche $(4 \mathrm{t}$ de $\mathrm{MS}$ environ en 1984) et à peu près équivalente pour les 2 espèces. L'effet de l'azote est très atténué par la sécheresse, notamment en début de croissance en 1985 et il est totalement annihilé en 1984.

Le tableau 1 permet de synthétiser les effets combinés de la sécheresse et du manque d'azote au moyen du modèle de croissance présenté dans la note précédente (LEMAIRE \& DENOIX, 1987) :

$$
\text { MS }=b(j-a)
$$

dans lequel $\mathbf{j}$ est le nombre de jours après la coupe, $b$ représente la vitesse de croissance moyenne en matière sèche durant la phase linéaire de la courbe de croissance et a le temps de latence apparent pour le redémarrage après la coupe. Nous avions montré précédemment qu'il existait une corrélation très étroite entre les 2 coefficients $a$ et $b$ et de ce fait nous avons choisi de caractériser la rapidité de redémarrage après la coupe par le nombre de jours a' nécessaires pour obtenir une production de $1000 \mathrm{~kg}$ de MS, sachant qu'au-delà la croissance est pratiquement linéaire. Sur le tableau 1, les effets des différents traitements eau $x$ azote sur la croissance en matière sèche sont analysés en fonction de 3 critères :

- rapidité de redémarrage : $\mathrm{a}^{\prime}$,

- vitesse de croissance : $b$,

- production obtenue après 50 jours de repousse : MS $50 \mathrm{j}$.

La rapidité de redémarrage en irrigué est très affectée lorsque le niveau de nutrition azotée est très limitant $(0 \mathrm{~N}=30$ à $40 \mathrm{j})$; par contre, il n'y a plus d'effet entre les niveaux $60 \mathrm{~N}$ et $120 \mathrm{~N}$ (de 10 à $20 \mathrm{j}$ selon les années). Le dactyle semble toujours légèrement plus lent que la fétuque au redémarrage. C'est en 1981 que la repousse a été la plus rapide $(9$ à $10 \mathrm{j}$ seulement); ceci peut être lié au climat relativement pluvieux du début juillet qui aurait permis une élongation maximale des feuilles après la coupe en maintenant le potentiel de l'eau dans les feuilles à un niveau très haut (LEAFE et al., 1977).

Les sécheresses modérées de 1979 et 80 n'ont pas retardé la repousse mais ont uniquement diminué les vitesses de croissance ultérieures, le déficit hydrique s'étant, ces années-là, installé tardivement et progressivement. En 1984, le retard dans le départ en croissance a été beaucoup plus sensible : les réserves hydriques du sol étaient déjà en grande partie épuisées au moment de la coupe et seule une irrigation de $25 \mathrm{~mm}$
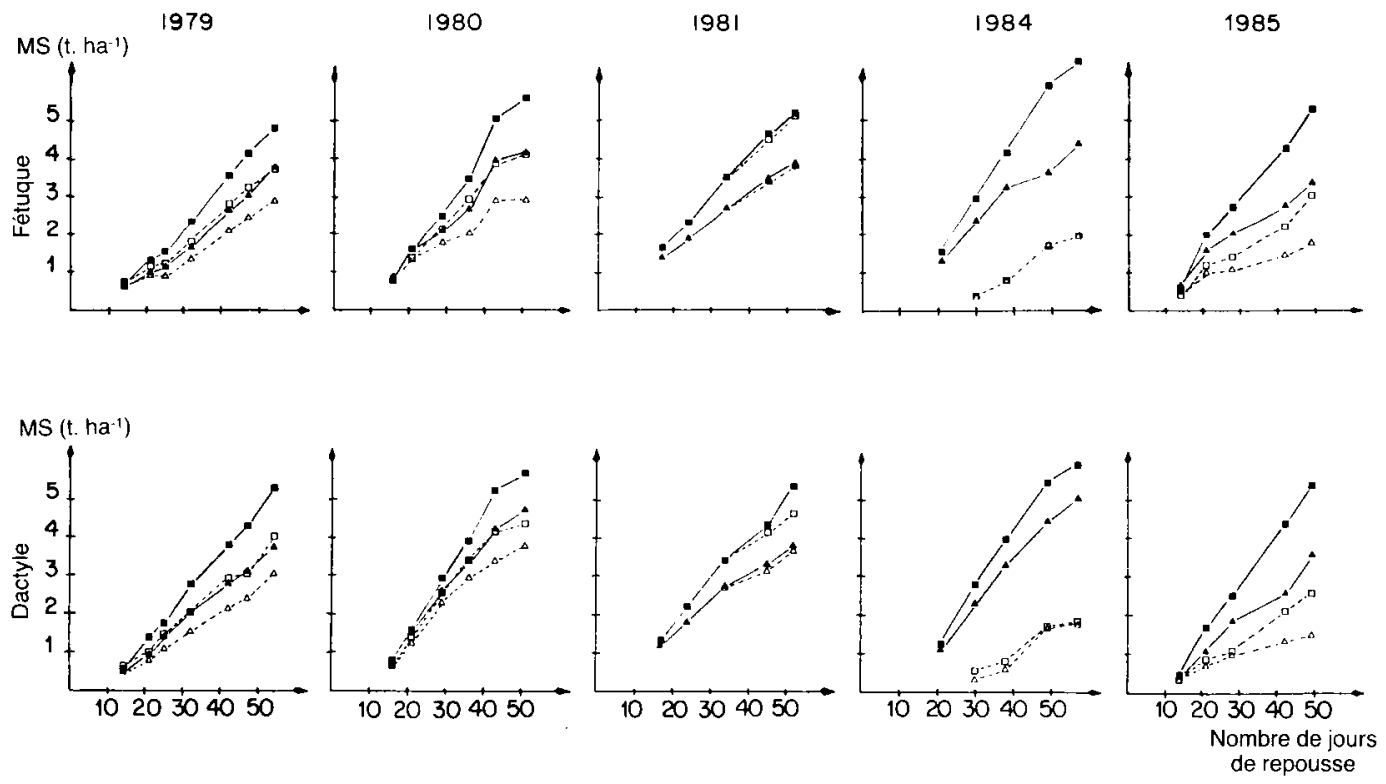

Figure 1

Courbes de croissance en matière sèche après une coupe de fin de printemps, a) Fétuque élevée cv. Clarine, b) Dactyle cv. Floreal, pour différentes années en fonction de la fertilisation azotée $(\square$ : $120 \mathrm{~kg} \mathrm{~N} ; \Delta \triangle: 60 \mathrm{~kg} \mathrm{~N}$ ), en condition sèche ( $\mathrm{L}, \triangle$ ) et en condition irriguée $(\mathbf{\square}, \mathbf{\Delta})$.
Summer regrowth curves of tall fescue $c v$. Clarine (a) and cocksfoot $c v$. Floreal (b) following a late spring cut for different years and two levels of nitrogen application $(\Delta \triangle: 60 \mathrm{~kg} \mathrm{~N} ; \square \square 120 \mathrm{~kg} \mathrm{~N})$, and for irrigated $(\triangle \square)$ or non irrigated plots $(\triangle \square)$. 
TABLEAU 1

Paramètres de l'équation $M S=b(J-a)$ décrivant la croissance en matière sèche au cours de la repousse estivale (1979-1985).

avec $b=$ vitesse de croissance $\mathrm{kg} \mathrm{MS} \mathrm{ha} \mathrm{a}^{-1} j^{-1}$

$J \quad=$ nombre de jours après la coupe

$a=$ = temps de latence apparent en jours

MS $50 j=$ quantité de matière sèche produite à $J=50$

= nombre de jours pour obtenir $M S=1000 \mathrm{~kg} \mathrm{ha}^{-1}$

Parameters of the equation $D M=b(J-a)$, which represents dry matter growth after cutting (1979-1985).

b = growth rate ( $\mathrm{kg} \mathrm{DM} \mathrm{ha} \mathrm{h}^{-1}$ day $^{-1}$ )

$J \quad=$ number of days after cutting

a = apparent delay of regrowth (days)

MS $50 j=$ dry matter yield after 50 days of regrowth

$a^{\prime} \quad=$ number of days for reaching $1000 \mathrm{~kg} \mathrm{DM} \mathrm{ha} \mathrm{a}^{-l}$.

\begin{tabular}{|c|c|c|c|c|c|c|}
\hline & 1979 & $\begin{array}{c}\mathrm{M} \\
\mathrm{kg} / \mathrm{ha}\end{array}$ & $\begin{array}{c}\mathrm{b} \\
\mathrm{kg} / \mathrm{ha} / \mathrm{j}\end{array}$ & $\begin{array}{l}\mathrm{a} \\
\mathrm{j}\end{array}$ & $\begin{array}{l}\mathrm{MS} \\
50 \mathrm{j} \\
\mathrm{t} / \mathrm{ha}\end{array}$ & $\begin{array}{l}\mathrm{a}^{\prime} \\
\mathrm{j}\end{array}$ \\
\hline $\begin{array}{l}\mathrm{F} \\
\hat{\mathrm{E}} \\
\mathrm{T}\end{array}$ & IRR & $\begin{array}{r}0 \\
60 \\
120\end{array}$ & $\begin{array}{r}47 \\
81 \\
107\end{array}$ & $\begin{array}{l}9,5 \\
8,8 \\
9,1\end{array}$ & $\begin{array}{l}1,90 \\
3,30 \\
4,40\end{array}$ & $\begin{array}{l}31 \\
21 \\
18\end{array}$ \\
\hline $\begin{array}{l}Q \\
\mathrm{U} \\
\mathrm{E}\end{array}$ & SEC & $\begin{array}{r}0 \\
60 \\
120\end{array}$ & $\begin{array}{l}34 \\
59 \\
79\end{array}$ & $\begin{array}{l}8,0 \\
6,4 \\
7,0\end{array}$ & $\begin{array}{l}1,40 \\
2,60 \\
3,40\end{array}$ & $\begin{array}{l}37 \\
23 \\
20\end{array}$ \\
\hline $\begin{array}{l}\mathrm{D} \\
\mathrm{A} \\
\mathrm{C}\end{array}$ & IRR & $\begin{array}{r}0 \\
60 \\
120\end{array}$ & $\begin{array}{r}39 \\
87 \\
118\end{array}$ & $\begin{array}{l}7,4 \\
8,1 \\
9,7\end{array}$ & $\begin{array}{l}1,70 \\
3,40 \\
4,70\end{array}$ & $\begin{array}{l}33 \\
20 \\
18\end{array}$ \\
\hline $\begin{array}{l}\mathrm{Y} \\
\mathrm{L} \\
\mathrm{E}\end{array}$ & SEC & $\begin{array}{r}0 \\
60 \\
120\end{array}$ & $\begin{array}{l}30 \\
63 \\
83\end{array}$ & $\begin{array}{l}6,8 \\
7,3 \\
7,5\end{array}$ & $\begin{array}{l}1,30 \\
2,70 \\
3,50\end{array}$ & $\begin{array}{l}40 \\
23 \\
20\end{array}$ \\
\hline
\end{tabular}

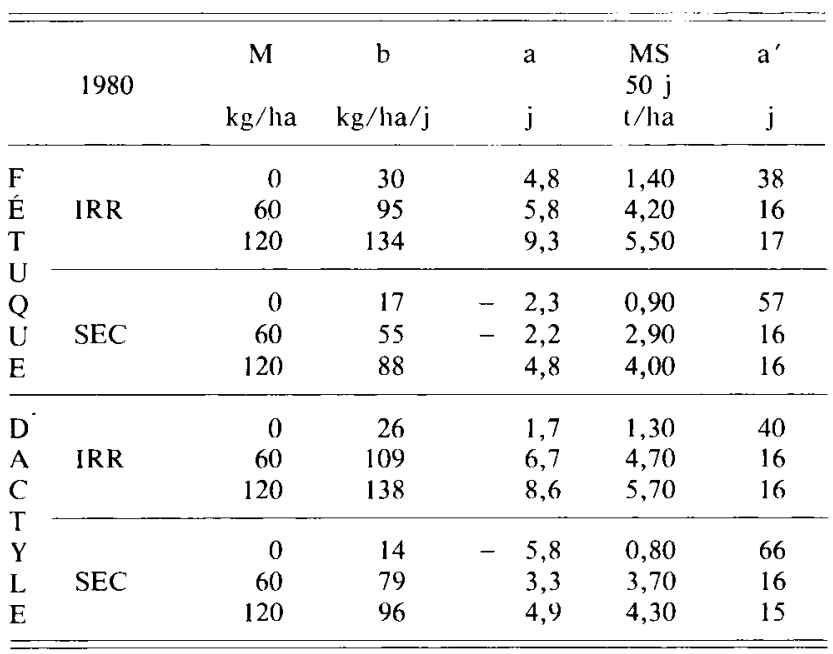

\begin{tabular}{|c|c|c|c|c|c|c|}
\hline & \multirow{2}{*}{1981} & $\mathbf{M}$ & b & $\mathrm{a}$ & MS & $a^{\prime}$ \\
\hline & & $\mathrm{kg} / \mathrm{l} \mathrm{a}$ & $\mathrm{kg} / \mathrm{ha} / \mathrm{j}$ & $\mathrm{j}$ & t/ha & $\mathrm{j}$ \\
\hline$F$ & \multirow{3}{*}{ IRR } & 0 & 31 & 5,1 & 1,70 & 27 \\
\hline$\hat{\mathrm{E}}$ & & 60 & 67 & $-4,5$ & 3,70 & 10 \\
\hline $\mathrm{T}$ & & 120 & 103 & 0,7 & 5,00 & 10 \\
\hline $\mathrm{Q}$ & \multirow{3}{*}{ SEC } & 0 & 22 & $-16,7$ & 1,40 & 29 \\
\hline U & & 60 & 65 & $-6,5$ & 3,70 & 9 \\
\hline E & & 120 & 92 & $-2,6$ & 4,80 & 8 \\
\hline D & \multirow{3}{*}{ IRR } & 0 & 22 & 8,9 & 1,30 & 37 \\
\hline A & & 60 & 67 & 3,2 & 3,60 & 12 \\
\hline C & & 120 & 110 & 4,2 & 5,10 & 13 \\
\hline$Y$ & \multirow{3}{*}{$\mathrm{SEC}$} & 0 & 14 & $-26,4$ & 1,10 & 45 \\
\hline $\mathrm{L}$ & & 60 & 60 & $-6,2$ & 3,40 & 10 \\
\hline E & & 120 & 82 & 3,0 & 4,40 & 9 \\
\hline
\end{tabular}

\begin{tabular}{|c|c|c|c|c|c|c|}
\hline & 1984 & $\begin{array}{c}M \\
\mathrm{~kg} / \mathrm{la} a\end{array}$ & $\begin{array}{c}\mathrm{b} \\
\mathrm{kg} / \mathrm{l} \mathrm{a} / \mathrm{j}\end{array}$ & $\begin{array}{l}\mathrm{a} \\
\mathrm{j}\end{array}$ & $\begin{array}{l}\text { MS } \\
50 \mathrm{j} \\
\mathrm{t} / \mathrm{ha}\end{array}$ & $\begin{array}{l}a^{\prime} \\
j\end{array}$ \\
\hline $\begin{array}{l}\mathrm{F} \\
\dot{\mathrm{E}} \\
\mathrm{T}\end{array}$ & IRR & $\begin{array}{r}60 \\
120\end{array}$ & $\begin{array}{r}82 \\
155\end{array}$ & $\begin{array}{r}2,4 \\
11,0\end{array}$ & $\begin{array}{l}3,90 \\
6,00\end{array}$ & $\begin{array}{l}15 \\
17\end{array}$ \\
\hline $\begin{array}{l}\mathrm{Q} \\
\mathrm{U} \\
\mathrm{E}\end{array}$ & $\mathrm{SEC}$ & $\begin{array}{r}60 \\
120\end{array}$ & $\begin{array}{l}63 \\
64\end{array}$ & $\begin{array}{l}24,3 \\
24,5\end{array}$ & $\begin{array}{l}1,60 \\
1,60\end{array}$ & $\begin{array}{l}40 \\
40\end{array}$ \\
\hline $\begin{array}{l}\mathrm{D} \\
\mathrm{A} \\
\mathrm{C}\end{array}$ & IRR & $\begin{array}{r}60 \\
120\end{array}$ & $\begin{array}{l}110 \\
150\end{array}$ & $\begin{array}{r}9,6 \\
12,1\end{array}$ & $\begin{array}{l}4,40 \\
5,70\end{array}$ & $\begin{array}{l}19 \\
19\end{array}$ \\
\hline $\begin{array}{l}Y \\
\mathrm{I} \\
\mathrm{E}\end{array}$ & SEC & $\begin{array}{r}60 \\
120\end{array}$ & $\begin{array}{l}57 \\
58\end{array}$ & $\begin{array}{l}24,5 \\
22,9\end{array}$ & $\begin{array}{l}1,50 \\
1,60\end{array}$ & $\begin{array}{l}42 \\
40\end{array}$ \\
\hline & 1985 & $\begin{array}{c}\mathrm{M} \\
\mathrm{kg} / \mathrm{ha}\end{array}$ & $\begin{array}{c}\mathrm{b} \\
\mathrm{kg} / \mathrm{ha} / \mathrm{j}\end{array}$ & $\begin{array}{l}\mathrm{a} \\
\mathrm{j}\end{array}$ & $\begin{array}{l}\text { MS } \\
50 \mathrm{j} \\
\text { l/ha }\end{array}$ & $\begin{array}{l}a^{\prime} \\
j\end{array}$ \\
\hline $\begin{array}{l}\text { F } \\
\text { É } \\
T\end{array}$ & IRR & $\begin{array}{r}60 \\
120\end{array}$ & $\begin{array}{r}72 \\
127\end{array}$ & $\begin{array}{l}1,5 \\
7,2\end{array}$ & $\begin{array}{l}3,50 \\
5,40\end{array}$ & $\begin{array}{l}15 \\
15\end{array}$ \\
\hline $\begin{array}{l}\text { Q } \\
\text { U } \\
\text { E }\end{array}$ & SEC & $\begin{array}{r}60 \\
120\end{array}$ & $\begin{array}{l}34 \\
69\end{array}$ & $\begin{array}{r}-\quad 4,1 \\
6,4\end{array}$ & $\begin{array}{l}1,80 \\
3,00\end{array}$ & $\begin{array}{l}25 \\
21\end{array}$ \\
\hline $\begin{array}{l}\text { D } \\
\text { A } \\
\text { C }\end{array}$ & IRR & $\begin{array}{r}60 \\
120\end{array}$ & $\begin{array}{r}71 \\
137\end{array}$ & $\begin{array}{l}6,5 \\
9,8\end{array}$ & $\begin{array}{l}3,10 \\
5,50\end{array}$ & $\begin{array}{l}21 \\
17\end{array}$ \\
\hline $\begin{array}{l}\text { Y } \\
\text { L } \\
\text { E }\end{array}$ & SEC & $\begin{array}{r}60 \\
120\end{array}$ & $\begin{array}{l}32 \\
63\end{array}$ & $\begin{array}{l}0,0 \\
8,6\end{array}$ & $\begin{array}{l}1,60 \\
2,60\end{array}$ & $\begin{array}{l}31 \\
24\end{array}$ \\
\hline
\end{tabular}

a pu permettre un léger redémarrage. Ceci pose le problème de la sensibilité particulière de la prairie au déficit hydrique après une coupe qui se traduit par un ralentissement très important de l'élongation des feuilles même pour des déficits hydriques modérés (LEAFE et al., 1977).

\section{B. Etude des consommations d'eau}

Le tableau 2 nous donne, pour les différentes années et pour les $71^{\text {res }}$ semaines de repousse, les valeurs cumulées de l'ETP Penman et des consommations hydriques en sec (ETR) et en irrigué (ETM), toutes doses d'azote confondues.

On peut noter qu'il n'y a pas une parfaite concordance entre l'ETP estimée par la formule de Penman et l'ETM mesurée à la sonde à neutron : le rapport ETM/ETP est en général inférieur à 1 en début de repousse lorsque la surface foliaire est peu développée (WHITE \& BROWN, 1972) et il devient supérieur à 1 lorsque le couvert végétal est bien développé. Sur l'ensemble de la repousse ce rapport est voisin de 1 pour les années à ETP modérée (1980-81) ; par contre l'ETP Penman conduit à une forte sous-estimation de la consommation en eau en année à forte ETP (1984$85)$.

La figure 2 nous permet de mettre en évidence l'effet du niveau de nutrition azotée sur la consommation en eau. Ainsi en 1979 et en 1980, lorsque l'on 


\section{TABLEAU 2}

Evapotranspiration of non-irrigated (ETR) and irrigated plots guees (ETM) cumulee sur les 50 premiers jours de repousse. Comparaison avec l'ETP Penman.

Evapotranspiration of non-irrigated (ETR) and irrigated plots (ETM) cumulated for 50 day's after cultung and comparison with Penman (ETP).

\begin{tabular}{lccccc}
\hline & Annee & ETP & ETR & ETM & ETR/ETM \\
\hline $\mathrm{F}$ & & & & & \\
$\mathrm{E}$ & 1979 & 190 & 134 & 162 & 0,83 \\
$\mathrm{~T}$ & 1980 & 153 & 133 & 168 & 0,80 \\
$\mathrm{U}$ & 1981 & 168 & 170 & 170 & 1,00 \\
$\mathrm{Q}$ & 1984 & 206 & 147 & 233 & 0,63 \\
$\mathrm{U}$ & 1985 & 203 & 208 & 254 & 0,82 \\
$\mathrm{E}$ & & & & & \\
\hline $\mathrm{D}$ & & & & & \\
$\mathrm{A}$ & 1979 & 190 & 132 & 166 & 0,80 \\
$\mathrm{C}$ & 1980 & 153 & 135 & 167 & 0,80 \\
$\mathrm{~T}$ & 1981 & 168 & 158 & 163 & 0,97 \\
$\mathrm{Y}$ & 1984 & 206 & 128 & 216 & 0,59 \\
$\mathrm{~L}$ & 1985 & 203 & 167 & 244 & 0,68 \\
$\mathrm{E}$ & & & & & \\
\hline \hline
\end{tabular}
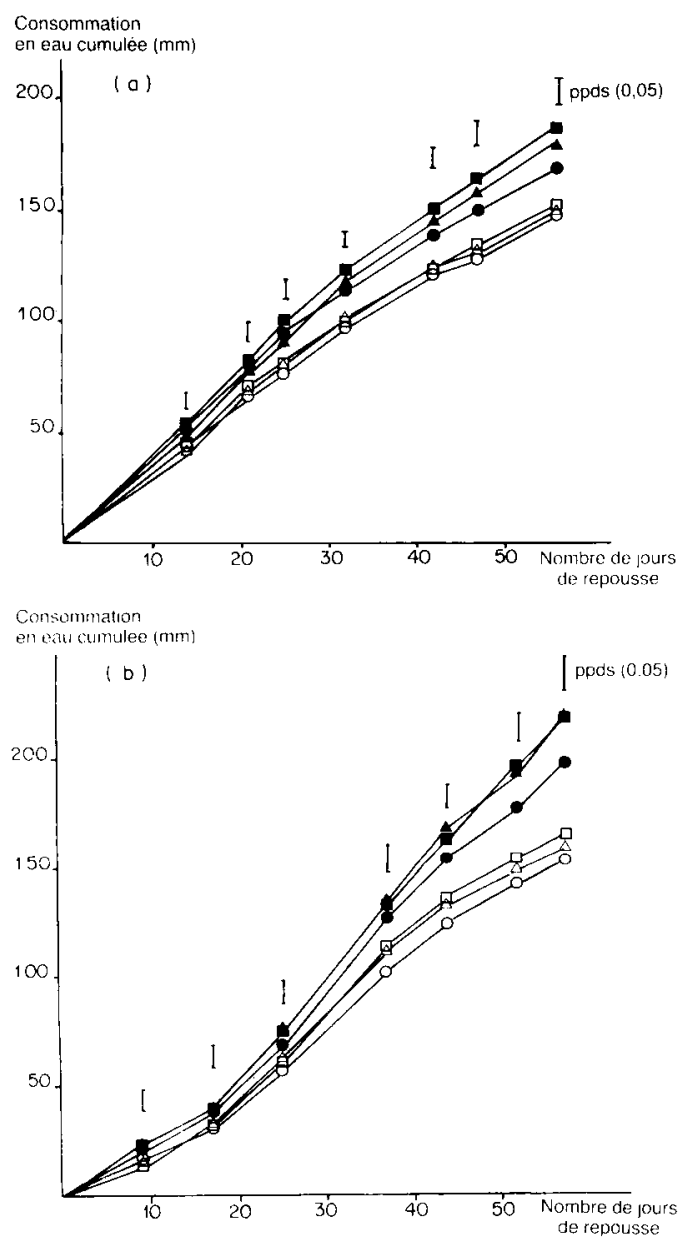

Figure 2

Consommation en eau cumulée au cours de la repousse de la Fétuque élevée, en 1979 (a) et en $1980(b)$, pour les différents traitements azotés $1 \bullet \bigcirc: 0 \mathrm{~N} ; \Delta \triangle: 60 \mathrm{~N} ; \square \square: 120 \mathrm{~N}$, en irrigué

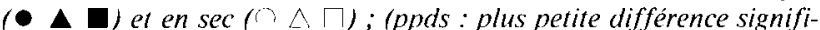
cative).

Cumulated water consumption during regrowth of tall fescue in $1979(a)$ and $1980(b)$, in irrigated $(\bullet \mathbf{\Delta})$ and non-irrigated plots (1. $\therefore$ i. 1$)$, and for different levels of nitrogen application : $0 \mathrm{~N}$

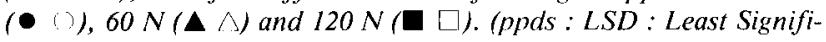
cant Difference). passe de 0 à $120 \mathrm{~kg}$ d'azote, la consommation en eau des parcelles irriguées augmente de $20 \mathrm{~mm}$, soit 11 p. 100 environ. En sec, cette augmentation de consommation est beaucoup plus faible et même négligeable. Ceci correspond aux résultats de LAUENROTH \& SIMS (1976) qui obtiennent une augmentation de 15 p. 100 de consommation d'eau par un apport de $180 \mathrm{~kg}$ d'azote sur une prairie naturelle.

\section{Relation entre croissance en matière sèche et con- sommation d'eau}

Le rapport des consommations en eau en sec et en irrigué : ETR/ETM peut nous servir à caractériser le niveau de satisfaction des besoins en eau du couvert végétal. Ce rapport peut être mis en relation avec le rapport des productions de matière sèche correspondantes : MS (ETR)/MS (ETM), selon le modèle développé par ROBELIN (1969).

Sur le tableau 3, on retrouve bien le classement des différentes années que nous avions réalisé au vu des croissances en matière sèche :

$\begin{array}{lllll}1981 \text { absence de sécheresse } & \text { ETR/ETM } & 1 & \\ 1979-80 & \text { sécheresse modérée } & : \text { ETR/ETM } & 0,80 & \\ 1984-85 \text { sécheresse forte } & : \text { ETR/ETM } & 0,60 & 0,80 .\end{array}$

On retrouve également une bonne concordance entre les 2 espèces avec une exception toutefois en 1985 où l'intensité de la sécheresse sur dactyle $(\mathrm{ETR} / \mathrm{ETM}=0,68)$ a été plus prononcée que sur la fétuque élevée $(E T R / E T M=0,82)$.

La figure 3 permet de visualiser la relation existant entre le déficit relatif de consommation d'eau et le déficit relatif de production, pour les deux espèces. Pour l'ensemble des années, les points correspondant aux différents niveaux $\mathrm{N}$ ne s'écartent pas significativement entre eux. Pour plus de clarté, sur la figure 3 ne sont représentés que les points moyens des 2 doses $60 \mathrm{~N}$ et $120 \mathrm{~N}$ (seuls traitements communs à l'ensemble des années).

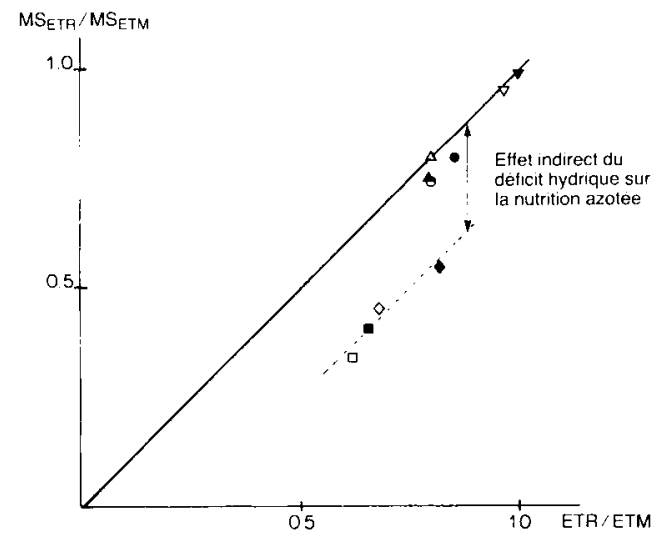

Figure 3

Relation entre l'indice de production MS (ETR)/MS (ETM) et l'indice de consommation en eau ETR/ETM pour la Fétuque (1979: •, 1980: $\mathbf{\Delta}, 1981: \mathbf{\nabla}, 1984: \mathbf{0}, 1985: \bullet)$ et le Dactyle (1979: $, 1980: \triangle, 1981: \nabla, 1984: \sqcap, 1985: \diamond)$.

Relationship between Relative Dry Matter production (DM ETR/ DM ETM) and Relative Water Consumption ETR/ETM of nonirrigated plots compared to irrigated plots, for tall fescue 11979. $\bullet, 1980: \mathbf{\Lambda}, 1981: \mathbf{\nabla}, 1984: \mathbf{\square}, 1985: \bullet)$, and cocksfoot (1979: ( $1980: \wedge, 1981: \nabla, 1984: \sqcap, 1985: \diamond)$ 
Pour les 3 années $1979-80$ et 81 , à sécheresse nulle et modérée, les points s'alignent approximativement sur la bissectrice, conformément aux résultats obtenus par MARTY et al. (1977) à Toulouse sur la fétuque élevée et MERIAUX (1977) à Dijon sur fétuque élevée et dactyle.

Par contre, en 1984 et 1985, on observe une diminution de production plus que proportionnelle à la diminution de consommation d'eau. Ainsi, en 1985, pour la fétuque élevée, à un niveau de sécheresse ETR/ETM approximativement équivalent à celui de 1979 et 1980 , on obtient une réduction de croissance nettement plus forte.

Ces résultats nous amènent à poser deux questions :

- Comment expliquer les résultats globaux de 1984 et 1985 qui mettent en évidence une sensibilité à la sécheresse beaucoup plus grande qu'en 1979 et 80 et que celle déterminée par des travaux précédents (MARTY \& PUECH, 1971 ; MARTY, 1977 ; MERIAUX, 1977) ?

- Comment expliquer qu'en 1985, placés dans le même sol et soumis au même climat, l'intensité de la sécheresse ait été plus faible sur fétuque élevée $(\mathrm{ETR} / \mathrm{ETM}=0,82)$ que sur dactyle $(\mathrm{ETR} / \mathrm{ETM}=$ $0,68)$ ?

\section{Effets de la sécheresse sur la nutrition azotée}

Une des hypothèses plausibles permettant d'expliquer l'amplification de l'effet de la sécheresse sur la croissance en matière sèche pourrait être fournie par les résultats de GARWOOD \& WILliaMS (1967a-b) qui signalent que le dessèchement des horizons superficiels riches en éléments minéraux et en azote en particulier, peut contribuer à diminuer la croissance de la prairie même si la consommation en eau dans les couches plus profondes permet de couvrir les besoins de la plante. Ainsi, dans les conditions d'une sécheresse plus marquée en 1984 et 1985 , à l'effet direct de la sécheresse sur la croissance en matière sèche a pu se superposer l'effet indirect d'une moins bonne disponibilité de l'azote minéral et/ou du phosphore et de la potasse.

Nous avions la possibilité de tester cette hypothèse au niveau de l'azote en comparant les courbes de dilution de l'azote $\mathrm{N} \%=\alpha(\mathrm{MS})^{-\beta}$ (Lemaire \& SALETTE, 1984) dans les 2 situations en sec et en irrigué. La figure 4 représente les courbes obtenues sur la fétuque élevée pour les années 1979 et 1985, années où l'intensité de la sécheresse était du même ordre $(E T R / E T M \simeq 0,80)$. Nous constatons qu'en 1979 la sécheresse n'a pas modifié la courbe de dilution et nous avons montré dans l'article précédent (LEMAIRE \& DENOIX, 1987) que ceci pouvait s'interpréter comme le signe d'une disponibilité équivalente de l'azote : seule la cinétique de dilution est ralentie du fait d'une croissance moins rapide. En 1985, au contraire, on obtient des courbes de dilution différentes, indiquant une déficience de nutrition azotée pour les parcelles non irriguées. Des résultats tout à fait similaires sont obtenus en 1984 et retrouvés également sur le dactyle. En revanche, en 1980, on confirme que la sécheresse, comme en 1979, n'a pas perturbé le niveau d'alimentation azotée.

On peut alors se demander pourquoi, pour une même intensité de la sécheresse exprimée par le rapport ETR/ETM, la nutrition azotée de la fétuque élevée non irriguée s'est trouvée affectée en 1985 alors qu'elle ne l'a pas été en $1979 \mathrm{ni}$ en 1980 . On peut expliquer cette différence de comportement par l'analyse du régime pluviométrique de cés 3 années. En 1979 et 1980, pour les 20 premiers jours de repousse, il y a eu respectivement 46 et $52 \mathrm{~mm}$ de fluie qui sont venus s'ajouter aux 5 à $10 \mathrm{~mm}$ apportés par irrigation après l'épandage d'azote, alors qu'en

TABLEAU 3

Evolution du déficit hydrique théorique $P$ - ETP ( $\mathrm{mm}$ ) par décade pour les différentes années.

Difference between rainfall $(P)$ and Potential Evapotranspiration (ETP in $\mathrm{mm}$ ) for each ten-day period over the different years.

\begin{tabular}{|c|c|c|c|c|c|c|c|c|}
\hline \multirow{2}{*}{ Année } & \multirow{2}{*}{$\begin{array}{c}\text { Déficit } \\
\text { initial } \\
\left(^{*}\right)\end{array}$} & & \multicolumn{3}{|c|}{ Juillet } & \multicolumn{2}{|c|}{ Août } & \multirow[b]{2}{*}{ Total } \\
\hline & & & $1-10$ & $10-20$ & $20-30$ & $1-10$ & $10-20$ & \\
\hline 1979 & -66 & $\begin{array}{c}\text { ETP } \\
\text { P } \\
P-\text { ETP }\end{array}$ & $\begin{array}{r}55,8 \\
0,0 \\
-\quad 55,8\end{array}$ & $\begin{array}{r}46,2 \\
47,0 \\
0,8\end{array}$ & $\begin{array}{r}48,2 \\
4,1 \\
-44,1\end{array}$ & $\begin{array}{r}38,0 \\
23,8 \\
-\quad 14,2\end{array}$ & $\begin{array}{r}27,4 \\
1,6 \\
-\quad 25,8\end{array}$ & $\begin{array}{r}216 \\
77 \\
-\quad 205\end{array}$ \\
\hline 1980 & -83 & $\begin{array}{c}\text { ETP } \\
\stackrel{P}{P}-\text { ETP }\end{array}$ & $\begin{array}{r}31,6 \\
15,6 \\
-\quad 16,0\end{array}$ & $\begin{array}{l}30,3 \\
41,8 \\
11,5\end{array}$ & $\begin{array}{r}44,8 \\
7,7 \\
-\quad 37,1\end{array}$ & $\begin{array}{r}38,7 \\
2,4 \\
-\quad 36,3\end{array}$ & $\begin{array}{r}30,3 \\
5,9 \\
-\quad 24,4\end{array}$ & $\begin{array}{r}176 \\
73 \\
-178\end{array}$ \\
\hline 1981 & -20 & $\begin{array}{c}\text { ETP } \\
\text { P } \\
P-\text { ETP }\end{array}$ & $\begin{array}{r}32,6 \\
37,0 \\
4,4\end{array}$ & $\begin{array}{r}34,7 \\
6,0 \\
-\quad 28,7\end{array}$ & $\begin{array}{r}45,7 \\
11,2 \\
-34,5\end{array}$ & $\begin{array}{r}33,5 \\
24,0 \\
-\quad 9,5\end{array}$ & $\begin{array}{r}40,1 \\
4,9 \\
-\quad 35,2\end{array}$ & $\begin{array}{r}187 \\
83 \\
-\quad 124\end{array}$ \\
\hline 1984 & -120 & $\begin{array}{c}\text { ETP } \\
\mathbf{P} \\
\mathrm{P}-\mathrm{ETP}\end{array}$ & $\begin{array}{r}52,3 \\
0,0 \\
-\quad 52,3\end{array}$ & $\begin{array}{r}44,9 \\
3,1 \\
-\quad 41,8\end{array}$ & $\begin{array}{r}49,7 \\
43,5 \\
-\quad 6,2\end{array}$ & $\begin{array}{r}30,8 \\
14,3 \\
-16,5\end{array}$ & $\begin{array}{r}38,4 \\
0,2 \\
-\quad 38,2\end{array}$ & $\begin{array}{r}216 \\
61 \\
-275\end{array}$ \\
\hline 1985 & -33 & $\begin{array}{c}\text { ETP } \\
P \\
\mathrm{P}-\mathrm{ETP}\end{array}$ & $\begin{array}{r}50,1 \\
12,2 \\
-37,9\end{array}$ & $\begin{array}{r}47,0 \\
4,2 \\
-\quad 42,8\end{array}$ & $\begin{array}{r}44,4 \\
30,0 \\
-\quad 14,4\end{array}$ & $\begin{array}{r}33,7 \\
8,4 \\
-\quad 25,3\end{array}$ & $\begin{array}{r}31,9 \\
4,6 \\
4,6\end{array}$ & $\begin{array}{r}207 \\
-\quad 59 \\
-\quad 153\end{array}$ \\
\hline
\end{tabular}

(*) Déficit mesuré du niveau des réserves hydriques par rapport à la capacité au clıamp. 
TABLEAU 4

Variation de l'efficience de l'eau consommée ( $\mathrm{kg} . \mathrm{MS} . \mathrm{mm}^{-1}$ ) en fonction du niveau de nutrition azotée (N). Variation of water use efficiency $\left(\mathrm{kg} . \mathrm{DM} . \mathrm{mm}^{-1}\right.$ ) according to the level of nitrogen nutrition $(N)$.

\begin{tabular}{|c|c|c|c|c|c|c|c|}
\hline & & \multicolumn{3}{|c|}{ Traitements secs } & \multicolumn{3}{|c|}{ Traitements irrigués } \\
\hline & & $0=\mathrm{N}$ & $60=N$ & $120=N$ & $0=\mathrm{N}$ & $60=N$ & $120=\mathrm{N}$ \\
\hline 1 & Fétuque & 10,0 & 18,7 & 24,5 & 12,9 & 19,1 & 25,9 \\
\hline \multicolumn{8}{|l|}{9} \\
\hline 9 & Dactyle & 10,3 & 18,2 & 23,1 & 10,9 & 18,7 & 25,8 \\
\hline 1 & Fétuque & 6,0 & 21,9 & 28,5 & 7,2 & 23,5 & 30,1 \\
\hline \multicolumn{8}{|l|}{9} \\
\hline \multicolumn{8}{|l|}{8} \\
\hline 0 & Dactyle & 5,5 & 25,2 & 30,6 & 7,6 & 25,2 & 31,3 \\
\hline
\end{tabular}

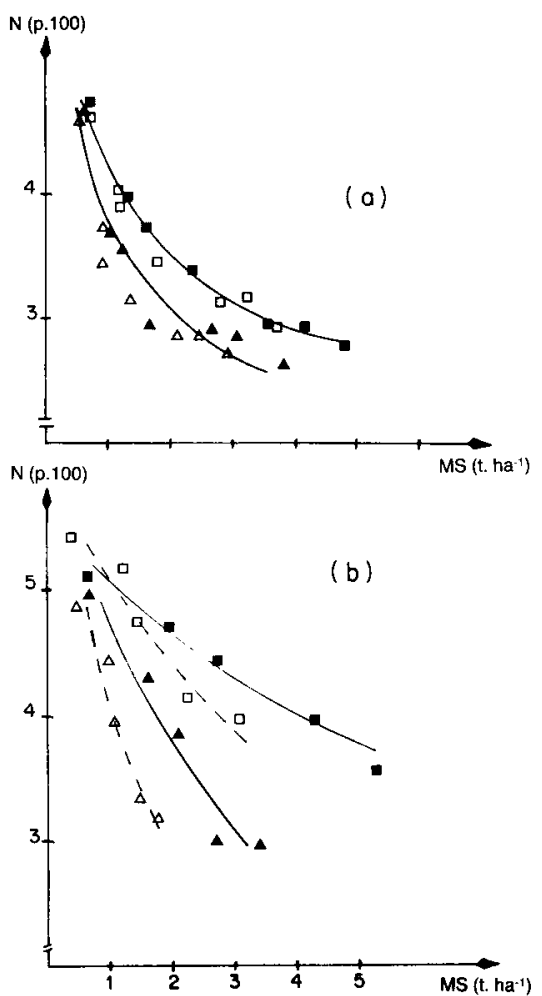

Figure 4

Relation de dilution de l'azote $N \%=\alpha(M S)^{-\beta}$ an cours de la repousse estivale de la Fétuque élevée a) : 1979, b) : 1985, en irrigué $(\Delta 60 \mathrm{~kg} \mathrm{~N} ; 120 \mathrm{~kg} \mathrm{~N})$ et en $\sec (\triangle 60 \mathrm{~kg} \mathrm{~N} ; \square 120 \mathrm{~kg} \mathrm{~N})$.

Relation of dilution of nitrogen during summer regrowth $N \%=\alpha$ $(D M)^{-\beta}$ for tall fescue in 1979 (a) and 1985 (b) for irrigated plots $(\Delta: 60 \mathrm{~kg} \mathrm{~N}, \mathbf{\square}: 120 \mathrm{~kg} \mathrm{~N})$ and non irrigated plots $(\triangle: 60 \mathrm{~kg} \mathrm{~N}$, $\square: 120 \mathrm{~kg} \mathrm{~N}$ ).

1985, pour cette même période, il n'y a eu que $12 \mathrm{~mm}$ de pluie. Or, les besoins en azote sont plus importants en début de repousse car ils correspondent à la mise en place de la surface foliaire pour l'interception du rayonnement. De plus, en 1985 , le déficit hydrique initial du sol était moins important $(-33 \mathrm{~mm})$ qu'en 1979 ( $-66 \mathrm{~mm}$ ) et qu'en 1980 ( $-83 \mathrm{~mm}$ ) (tabl. 3). L'ensemble de ces observations indique qu'en 1985 l'alimentation en eau en début de repousse s'est effectuée à partir des horizons plus profonds et moins riches en azote, alors qu'en 1979 et 1980 la réhumectation plus importante des horizons superficiels par la pluie a permis une meilleure disponibilité de l'azote.

\section{E. Efficience de l'eau consommée}

Cet effet secondaire de la sécheresse sur l'alimentation minérale se retrouve également sur les éléments $P$ et $\mathrm{K}$ (données non publiées). On confirme donc parfaitement les résultats de GARWOOD \& WILLIAMS $(1967 a-b)$ obtenus, comme à Lusignan, dans un sol à réserves hydriques profondes. Ceci explique que l'efficience de l'eau consommée dans les horizons profonds sont plus faibles que celle des horizons superficiels. En 1979, 80 et 81, l'efficience de l'eau n'est absolument pas modifiée par le niveau de l'alimentation hydrique et nous confirmons ainsi les résultats de MARTY \& PUECH (1971) et PUECH \& MAERTENS (1974) sur les graminées fourragères. Au contraire, en 1984 et 1985 il y a une forte diminution de l'efficience avec la sécheresse lorsque les plantes explorent les couches profondes du sol : 13 à $15 \mathrm{~kg}$ de MS par mm d'eau en sec et 22 à 25 en irrigué (tabl. 4).

Le manque d'azote diminue l'efficience de l'eau. Ceci est particulièrement mis en évidence dans le tableau 4 : avec $\mathrm{N}=0$ l'efficience est de $10 \mathrm{~kg}$ de $\mathrm{MS}$ par mm d'eau en 1979 et de 6 à 7 en 1980, alors qu'avec $\mathrm{N}=120$, pour les deux années, elle est de 26

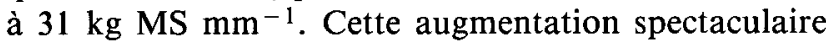

\section{TABLEAU 5}

Comparaison de l'efficience de l'eau consommée ( $\mathrm{kg} . \mathrm{MS} . \mathrm{mm}^{-1}$ ) en sec et en irrigué

Comparison of water use efficiency $\left(\mathrm{kg} . \mathrm{DM} . \mathrm{mm}^{-1}\right)$ of tall fescue and cocksfoot on irrigated and non-irrigated plots.

\begin{tabular}{cccc}
\hline \hline & Année & $\begin{array}{c}\text { Traitements } \\
\text { secs }\end{array}$ & $\begin{array}{c}\text { Traitements } \\
\text { irrigués }\end{array}$ \\
\hline F & & & \\
E & 1979 & 24,5 & 25,9 \\
T & 1980 & 28,5 & 30,1 \\
U & 1981 & 26,5 & 27,5 \\
Q & 1984 & 11,8 & 25,4 \\
U & 1985 & 14,2 & 20,9 \\
E & & & \\
\hline D & & & \\
A & 1979 & 23,1 & 25,8 \\
C & 1980 & 30,6 & 26,6 \\
T & 1981 & 26,1 & 25,3 \\
Y & 1984 & 13,4 & 22,0 \\
L & 1985 & 15,4 & \\
E & & & \\
\hline \hline
\end{tabular}


de l'efficience de l'eau avec la nutrition azotée est la résultante d'un effet très important sur la croissance et d'un effet relativement très modeste sur la consommation d'eau (fig. 2).

\section{F. Différence de comportement des deux espèces vis- à-vis de la sécheresse et du manque d'azote}

Sur l'ensemble des années étudiées, on a pu noter un comportement identique de la fétuque élevé cv. Clarine et du dactyle cv. Floreal vis-à-vis de la sécheresse, excepté en 1985 où, pour une ETM à peu près identique de $250 \mathrm{~mm}$, les 2 espèces ont eu des consommations en eau assez différentes en sec : $167 \mathrm{~mm}$ pour le dactyle, contre $208 \mathrm{~mm}$ pour la fétuque élevée (tabl. 2).

L'analyse des profils de dessèchement maximum du sol par les 2 cultures nous montre que, même lorsque les quantités d'eau extraites sont identiques, le comportement des 2 espèces est différent (fig. 5). Le dactyle extrait plus d'eau des horizons superficiels et a par contre une extraction réduite en profondeur par rapport à la fétuque élevée. En règle générale, lorsque le dessèchement du sol est peu prononcé (1979-80) les avantages respectifs de chacune des 2 espèces se compensent et leurs consommations globales d'eau du sol sont identiques. Par contre, en année à forte sécheresse (1984 et 1985) le dactyle est plus rapidement limité dans sa consommation hydrique du fait de l'assèchement total des horizons superficiels. En 1984, les réserves hydriques profondes avaient été largement entamées lors du printemps exceptionnellement sec et la fétuque élevée n'a pu profiter totalement de sa capacité d'extraction de l'eau en profondeur : la différence de consommation d'eau du sol entre les 2 espèces a été limitée à $20 \mathrm{~mm}$. En revanche, en 1985, après un printemps pluvieux, la fétuque élevée a pu extraire $40 \mathrm{~mm}$ d'eau de plus que le dactyle dans les horizons profonds du sol. Ce supplément d'eau consommée par la fétuque élevée ne s'est traduit que par un supplément de production de $400 \mathrm{~kg}$ de MS sur le traitement $120 \mathrm{~N}$, soit une efficience de l'eau de $10 \mathrm{~kg} \mathrm{~mm}^{-1}$, ce qui correspond en fait à l'efficience moyenne obtenue en absence d'apport d'azote.

Cette différence dans la modalité de consommation de l'eau du sol entre les 2 espèces est liée à la morpho-

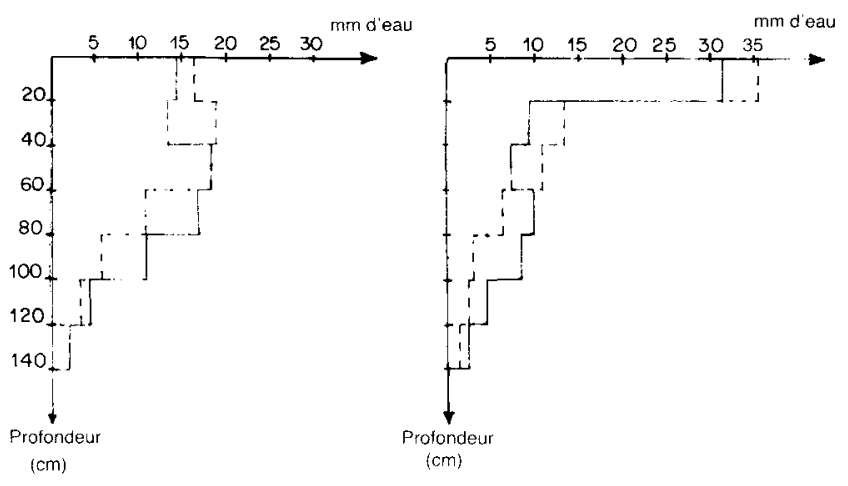

Figure 5

Profils de dessèchement maximum des horizons du sol sous Dactyle (--.---) et sous Fétuque (——).

Maximum soil water consumption from different depth of soil under cocksfoot (-------) and tall fescue (- logie de leurs systèmes racinaires : racines très ramifiées et très fines du dactyle, racines très longues, plus grosses et peu ramifiées de la fétuque élevée (données non publiées). De plus, ces caractéristiques du système racinaire ne sont pas sans conséquence sur la capacité de prélèvement de l'azote et des minéraux par les 2 peuplements.

Nous avons vu dans l'article précédent (LEMAIRE \& DENOIX, 1987) que lorsque la disponibilité de l'azote dans le sol n'était pas limitante, la quantité d'azote prélevée par les 2 espèces était équivalente. Ceci est confirmé par la figure 6 où l'on peut voir que, en condition irriguée, la relation entre la dynamique de prélèvement d'azote et la croissance en matière sèche est identique pour les 2 espèces pour $\mathrm{N} \mathrm{120}$. Par contre, à $\mathrm{N} 60$, on obtient, pour le dactyle, une courbe beaucoup plus proche de la courbe non limitante, ce qui est le signe d'une capacité plus grande pour cette espèce que pour la fétuque élevée à prélever l'azote du sol en situation de faible disponibilité.

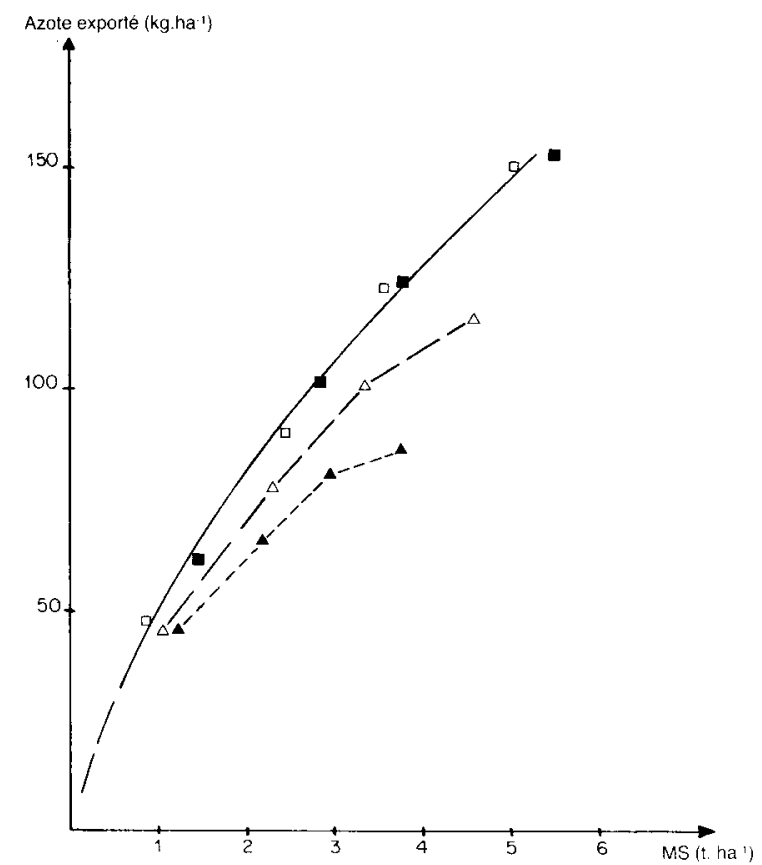

Figure 6

Dynamique de prélèvement d'azote en fonction de la croissance au cours de la repousse après un apport d'azote de $120 \mathrm{~kg} \mathrm{ha}^{-1}$ ( Fétuque, $\square:$ Dactyle) ou après un apport limité de $60 \mathrm{~kg} \mathrm{ha}^{-1}$ (। : Fétuque, $\triangle$ : Dactyle).

Dynamics of nitrogen uptake in relation with dry matter growth, with a high level of $N\left(120 \mathrm{~kg} \mathrm{ha}^{-1}\right): \mathbf{\square}$ tall fescue, $\square$ cocksfoot, or with a low level of $N\left(60 \mathrm{~kg} \mathrm{ha}^{-1}\right): \Delta$ tall fescue, $\triangle$ cocksfoot.

\section{CONCLUSION}

Les résultats obtenus sur 5 années aux conditions climatiques variées et à partir de 2 espèces ayant des capacités d'exploration du sol par les racines très différentes, nous ont permis d'analyser l'ensemble des mécanismes intervenant dans les interactions entre l'alimentation hydrique et la nutrition azotée. Il est en effet banal de montrer que l'irrigation améliore l'efficacité des apports d'azote et qu'inversement l'azote 
améliore l'efficacité de l'irrigation. Pour aller au-delà de cette simple constatation, la méthode dite « des courbes de dilution " nous a permis de séparer l'effet direct de la sécheresse de son effet indirect sur la disponibilité en azote du sol. Les résultats obtenus confirment que cette méthode représente un outil de diagnostic très précis du niveau réel de nutrition azotée d'un peuplement végétal dans une situation donnée.

La possibilité d'établissement d'une loi suffisamment générale permettant, à partir d'un potentiel de croissance déterminé, de connaître la réduction de production occasionnée par un niveau de déficit hydrique ou de déficit en azote se heurte à la difficulté de définir les conditions exactes dans lesquelles ces stress se produisent. Ainsi la connaissance du type d'enracinement, du profil pédologique, des propriétés hydrodynamiques du sol et de la répartition des élé- ments minéraux dans le profil est indispensable si l'on veut prévoir et quantifier l'effet d'un déficit hydrique ou azoté. L'effet de la répartition des éléments minéraux dans le profil est particulièrement important sur les prairies de longue durée où les éléments fertilisants sont apportés en surface sans possibilité de labours pour les enfouir en profondeur. La caractérisation de l'intensité d'une sécheresse par le rapport ETR/ETM apparaît insuffisante pour pouvoir prédire son effet sur la croissance d'une prairie. La prise en compte de l'humidité des horizons superficiels et du type d'enracinement des espèces considérées apparaît indispensable si l'on veut expliquer les effets indirects de la sécheresse sur l'alimentation minérale des peuplements prairiaux.

Reçu le 13 juin 1986 Accepté le 3 mars 1987.

\section{RÉFÉRENCES BIBLIOGRAPHIQUES}

Burzlaff D. F., Fick G. N., Rittenhouse L. R., 1968. Effect of nitrogen fertilization on certain factors of a western Nebraska range ecosystem. J. Range Manage., 21, 21-24.

Chesseron C., 1986. Contribution à l'étude des relations sol-plantes. Lupin et luzerne dans les terres rouges à châtaigniers du PoitouCharentes. Thèse, Faculte des Sciences, Univ. Poitiers, $142 \mathrm{p}$.

Colman R. L., Lazenby A., 1975. Effect of moisture on growth and nitrogen response by Lolium perenne. Plant and Soil, 42, 1-13.

Garwood E. A., Williams T. E., 1967a. Soil water use and growth of a grass sward. J. agric. Sci. Camb., 68, 281-292.

Garwood E. A., Williams T. E., 1967b. Growth, water use and nutrient uptake from the subsoil by grass swards. J. agric. Sci. Camb., 69, 125-130.

Lauenroth W. K., Sims P. L., 1976. Evapotranspiration from a short grass prairie subjected to water and nitrogen treatments. Water Resour. Res., 12, 437-442.

Leafe E. L., Jones M. B., Stiles W., 1977. The physiological effects of water stress on perennial ryegrass in the field. Proc. I3th Int. Grass. Congr., Leipzig, sect., 1-2, 165-184.

Lemaire G., Denoix A., 1987. Croissance estivale en matière sèche de peuplements de fétuque élevée (Festuca arundinacea Schreb.) et de dactyle (Dactylis glomerata L.) dans l'ouest de la France : I. Etude en conditions de nutrition azotée et d'alimentation hydrique non limitantes. Agronomie, 7 (6), 373-380.

Lemaire G., Roberge G., 1980. Mise au point d'un dispositif expérimental permettant de suivre la consommation hydrique d'une prairie au cours de la croissance. Ann. agron., 31 (4), 455-471.

Lemaire G., Salette J., 1984. Relation entre dynamique de croissance et dynamique de prélèvements d'azote pour un peuplement de graminées fourragères. I. Etude de l'effet du milieu. Agronomie, 4 (5), 423-430.

Marty J. R., 1977. Irrigation des fourrages : production et valorisation de l'eau de quelques espèces fourragères. Bull. tech. Inf., $\mathrm{n}^{\text {os }} 319-320,209-221$.

Marty J. R., Puech J., 1971. Efficience de l'eau en production fourragère. C. R. Acad. Agric., 57, 938-949.

Marty J. R., Bosc N., Hilaire A., 1977. Production comparée de quelques espèces fourragères en condition irriguée ou non. Efficience et valorisation de l'eau. Coûts de production. Fourrages, 70. $91-120$.

Meriaux S., 1977. Principes de conduite de l'irrigation des plantes fourragères. Fourrages, 70, 43-61.

Puech J., Maertens C., 1974. Efficience de l'eau consommée de quelques cultures placées dans différentes conditions écologiques. Agrochimica, 18, 223-230.

Robelin M., 1969. L'alimentation en eau des plantes fourragères. Fourrages, 38, 30-40.

Smika D. E., Haas H. J., Power J. F., 1965. Effects of moisture and nitrogen fertilizer on growth and water use by native grass. Agron. J., 57, 483-486.

Turner D. O., 1979. Nitrogen fertilization of orchardgrass pasture as influenced by irrigation. Washington State University, College of Agriculture Research Center, bull. 0881, 1-15.

White L. M., Brown J. H., 1972. Nitrogen fertilization and clipping effects on green needlegrass (Stipa viridula Trin.). II. Evapotranspiration, water-use efficiency and nitrogen recovery. Agron. J., 64, 487-490. 